\title{
ESTUDIO HISTOPATOLÓGICO COMPARATIVO DEL NERVIO FRÉNICO PROVENIENTE DE CADÁVERES CON ENFERMEDAD PULMONAR OBSTRUCTIVA CRÓNICA Y SIN ESTA CONDICIÓN
}

\author{
COMPARATIVE HISTOPATHOLOGICAL STUDY OF THE PHRENIC NERVE FROM CORPSES WITH \\ CHRONIC OBSTRUCTIVE PULMONARY DISEASE AND WITHOUT THIS CONDITION
}

Fernanda Mayara Santos-Santana', Byron Martins-Lucena², Tales Lyra de Oliveira, ${ }^{3,4}$, Tácio Nertan Cartaxo de Souza ${ }^{5}$, Lysien Ivania-Zambrano ${ }^{6}$, Ana Rose de Carvalho-Araujo7, Ana Lúcia de Gusmão-Freire ${ }^{8}$

\begin{abstract}
RESUMEN
Introducción: Los cambios funcionales resultantes de la evolución de la enfermedad pulmonar obstructiva crónica (EPOC) son progresivos e irreversibles, lo que provoca un aumento del trabajo del diafragma debido a la hiperinflación. Los nervios frénicos promueven la inervación del diafragma y pueden verse comprometidos en la condición de EPOC.Objetivo: Comparar la morfología de los nervios frénicos de los cadáveres con y sin EPOC, mediante microscopía óptica. Métodos: Estudio descriptivo, transversal y comparativo de muestras, tanto de los pulmones como de los nervios frénicos de 38 cadáveres, con diagnóstico de EPOC y sin esta condición. Las muestras de tejido fueron fijadas y procesadas por histología convencional con láminas histológicas de hematoxilina-eosina. Las muestras fueron compuestas por pacientes con EPOC (EPOC) y sin EPOC (CTR). Esta clasificación se realizó después del análisis histológico. Las láminas histológicas se analizaron mediante microscopía óptica por un patólogo, que fue un evaluador ciego al estudio. Resultados: De acuerdo con los criterios de inclusión, se incluyeron 31 cadáveres en el grupo de EPOC y 7 cadáveres en el grupo CTR. En el análisis de los nervios frénicos, 8 cadáveres, es decir 25,8\% del grupo de EPOC, tuvieron cambios histopatológicos como edema perineural $(75 \%, n=6)$, atrofia nerviosa $(12,5 \%$, $n=1)$ e infiltrado eosinofílico perineural $(12,5 \%, n=1)$. Conclusión: Los nervios frénicos de los cadáveres con EPOC tienen tendencia a alteraciones histopatológicas, siendo el edema perineural la principal modificación encontrada.
\end{abstract}

Palabras clave: Enfermedad pulmonar obstructiva crónica; Nervio frénico; Microscopía; Autopsia. (fuente: DeCS BIREME).

\begin{abstract}
Introduction: Functional changes resulting from the evolution of chronic obstructive pulmonary disease (COPD) are progressive and irreversible, causing increased diaphragm work due to pulmonary hyperinflation and airway obstruction. Phrenic nerves have promoted innervation of the diaphragm and may have been compromised in COPD condition. Objective: To compare the morphology of the phrenic nerves of the cadavers with COPD and without COPD by optical microscopy. Methods: An exploratory descriptive studio conducted on the Death Verification Service in Alagoas. Pulmonary and phrenic nerve biopsies will be bilaterally taken from the 38 cadavers after a necropsy with the diagnosis of COPD. Tissue samples were fixed and processed by conventional histology for hematoxylin-eosin (HE) histological slides. Biopsies are divided into experimental groups, one composed by patients with COPD and the other with patients without COPD (control - CTR). This classification was realized after the histological analysis, when typical halls of COPD were found. Histological slides were analyzed by optical microscopy by a pathologist, who was able to assess the study. Results: According to the inclusion and exclusion criteria of the study, if it includes 38 cadavers in the initial evaluation, of which 31 are included in the COPD group and 7 in the CTR group. In the analysis of the phrenic nerves, 8 cadavers, $25.8 \%$, of the COPD group had histopathological changes: perineural edema $(75 \%, n=6)$, nervous atrophy $(12.5 \%, n=1)$ and perineural eosinophilic infiltrate $(12.5 \%, n=1)$. The CTR group does not present histopathological alterations of the phrenic nerves. Conclusion: Given the hallmarks of the biopsies performed on the phrenic nerves of the corpses with COPD, we can infer that there is a tendency for nerve alteration, with perineural edema, to be the major modification found.
\end{abstract}

Key words: Chronic obstructive pulmonary disease; Phrenic nerve; Microscopy; Autopsy. (source: MeSH NLM).

\footnotetext{
${ }^{1}$ Faculdade de Medicina, Universidade Federal de Alagoas, Maceió - Brasil.

${ }^{2}$ Prefeitura de Maceió, Maceió - Brasil.

${ }^{3}$ Faculdade de Medicina, Universidade Municipal de São Caetano do Sul, São Paulo - Brasil.

${ }_{4}^{4}$ Unidade de Hipertensão, Instituto do Coração, Faculdade de Medicina, Universidade de São Paulo, São Paulo - Brasil.

5 Instituto de Ciências Biológicas e da Saúde, Universidade Federal de Alagoas, Maceió - Brasil.

${ }^{6}$ Departamento de Ciencias Morfológicas, Facultad de Ciencias Médicas. Universidad Nacional Autónoma de Honduras, Tegucigalpa - Honduras.

7 Serviço de Verificação de Óbitos, Universidade Estadual de Ciências da Saúde de Alagoas, Maceió - Brasil.

${ }^{8}$ Faculdade de Fisioterapia, Universidade Estadual de Ciências da Saúde de Alagoas, Maceió - Brasil.

Citar como: Fernanda Mayara Santos-Santana, Byron Martins-Lucena, Tales Lyra de Oliveira, Tácio Nertan Cartaxo de Souza, Lysien Ivania-Zambrano, Ana Rose de Carvalho-Araujo, Ana Lúcia de Gusmão-Freire. Estudio histopatológico comparativo del nervio frénico proveniente de cadáveres con enfermedad pulmonar obstructiva crónica y sin esta condición. Rev. Fac. Med. Hum. Abril 2020; 20(2):209-215. DOI 10.25176/RFMH.v20i2.2785
} 


\section{INTRODUCCIÓN}

La enfermedad pulmonar obstructiva crónica (EPOC) se considera la cuarta causa de muerte en el mundo y su progresión a 2020 es catastrófica, ya que puede ocupar la tercera posición en este ranking ${ }^{(1,2)}$. El principal factor de riesgo para la EPOC es fumar, pero otras exposiciones ambientales como la exposición a la biomasa y la contaminación del aire, pueden contribuir. Además de la exposición, algunos factores intrínsecos predisponen a los individuos a desarrollar EPOC, como anomalías genéticas, desarrollo anormal de los pulmones y envejecimiento acelerado ${ }^{(3)}$. La EPOC promueve la hipersecreción pulmonar, el edema de las vías respiratorias y los espasmos bronquiales que causan obstrucción del flujo de aire y aumentan la resistencia pulmonar. Durante la exacerbación de la EPOC, la inspiración se vuelve superficial y rápida, mientras que la exhalación se prolonga, haciendo imposible ventilar adecuadamente el aire de los pulmones, lo que lleva a una hiperinflación pulmonar ${ }^{(4)}$.

El músculo respiratorio principal es el diafragma, que proporciona ventilación pulmonar al elevar los últimos seis pares de costillas y esternón, aumentando el diámetro transverso y anteroposterior de la caja torácica, y a medida que la exacerbación de la EPOC se hace frecuente, se producen cambios en la mecánica ventilatoria, específicamente en la reducción de las cúpulas hemi diafragmáticas, lo que produce una presión abdominal inferior, lo que reduce la capacidad del diafragma para generar presión inspiratoria y menos expansión del tórax. Estos cambios promueven el acortamiento de los músculos ventilatorios, generando una desventaja mecánica para satisfacer las necesidades de la demanda respiratoria ${ }^{(5)}$.

Los cambios funcionales resultantes de la evolución de la EPOC son progresivos e irreversibles, lo que provoca un aumento constante del trabajo muscular ventilatorio debido a la hiperinflación pulmonar y la obstrucción del flujo de aire, lo que promueve la fatiga y el agotamiento del diafragma ${ }^{(6)}$.

El nervio frénico, que se origina a partir de las raíces nerviosas cervicales $\mathrm{C} 3-\mathrm{C} 5$, desempeña un papel importante en el proceso de ventilación, ya que es responsable de la inervación del diafragma ${ }^{(6)}$. La inervación periférica consiste básicamente en ganglios y nervios. El nervio frénico está compuesto de haces de fibras rodeados por una capa, llamada vaina de mielina. La vaina de mielina tiene un diámetro de aproximadamente $1 \mu \mathrm{m}$, que consiste en una membrana lipídica rica en glicofosfolípidos y colesterol que cubre los axones, facilitando la transmisión de los impulsos nerviosos ${ }^{(7)}$. Las células gliales son numerosas en el sistema nervioso y pueden clasificarse según sus propiedades funcionales y su ubicación: células de Schwann, ubicadas en el sistema nervioso periférico (SNP), y oligodendrocitos, ubicadas en el sistema nervioso central (SNC). Las células de Schwann y los oligodendrocitos rodean los axones formando varias capas $^{(8)}$.

La vaina de mielina actúa como un aislante eléctrico, promoviendo un mayor impulso nervioso porque causa saltos de despolarización a lo largo del axón a través de los nódulos de Ranvier. El grosor de la vaina de mielina, el diámetro del axón, la cantidad y la distancia entre los nódulos de Ranvier, la estructura nodal y la composición molecular de los canales iónicos, influyen en la velocidad de conducción de los impulsos nerviosos que pueden ser hasta 100 veces más rápidos ${ }^{(7)}$. Existe evidencia de que los nervios frénicos pueden verse comprometidos en la EPOC debido a cambios irreversibles en la mecánica ventilatoria debido fundamentalmente a cambios en el diafragma $a^{(9,10)}$.

El objetivo de este estudio fue comparar la morfología de los nervios frénicos de los cadáveres con EPOC y sin EPOC mediante microscopía óptica.

\section{MÉTODOS}

\section{Diseño}

Se realizó un estudio descriptivo exploratorio en el año 2012. Se tomaron muestras de los pulmones y nervios frénicos de 38 cadáveres sometidos a autopsia con diagnóstico de EPOC $(n=31)$ y sin esta condición $(n=7)$ en el servicio de verificación de muerte (SVO) de la Universidad Estatal de Ciencias de la Salud de Alagoas (UNCISAL). Las muestras se dividieron en dos grupos: 1) grupo con EPOC (EPOC) y 2) grupo sin EPOC (CTR). Es de destacar que los grupos fueron confirmados después del análisis histológico, respetando los criterios de inclusión y exclusión.

El formulario del consentimiento informado (ICF), que se adjunta al protocolo general del SVO, fue firmado por el miembro de la familia responsable del cadáver.

Esta investigación fue aprobada por el Comité de Ética en Investigación (CEP) de la Universidad Estatal de Ciencias de la Salud de Alagoas (UNCISAL).

Los criterios de exclusión fueron cadáveres con ausencia de lóbulo o pulmón debido a cirugía o malformación congénita, que se sometieron a cirugía cardíaca o que tuvieron algunas comorbilidades específicas, como enfermedad neuromuscular, enfermedad neurodegenerativa, malformación estructural del hueso torácico, enfermedad neurológica e historial de traumatismo (traumatismo craneal - TBI o traumatismo de la médula espinal - TRM). 
Inicialmente, se confirmóla información sobre el cadáver contenido en el formulario desarrollado por familiar en el SVO, que observó el historial de tabaquismo como uno de los principales criterios para la inclusión en el grupo con EPOC, el cual se confirmaría por la presencia asociada de algunos cambios histopatológicos específicos. Por el contrario, ningún cadáver incluido en el grupo de CTR tenía un historial de tabaquismo.

\section{Muestras frénicas y pulmonares}

El material para el análisis se recogió mediante el procedimiento de necropsia estándar realizado en el SVO, en el que se realizó un corte desde el manubrio hasta la cicatriz umbilical (Figura 1. A) a través del cual se accedió a las estructuras torácicas desde las que se accedió. Se tomaron muestras bilaterales de los ápices pulmonares (Figura 1. B) y de los nervios frénicos, localizados en las regiones posterolaterales del pericardio (Figura 1. C). Las muestras de ápice pulmonar sirvieron para confirmar la presencia o ausencia de antracosis, engrosamiento septal y bullas enfisematosas, que pueden ser resultados de la EPOC.
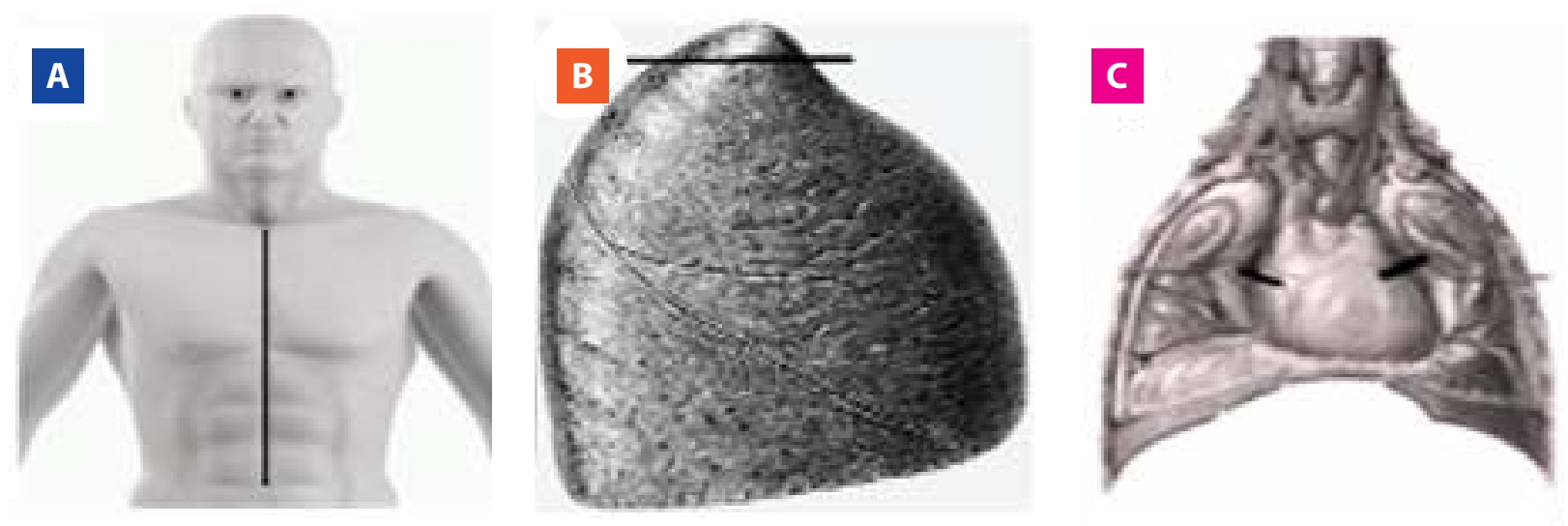

Figura 1. Procedimiento de necropsia estándar realizado. A) Sección de la región desde el manubrio hasta la cicatriz umbilical para realizar el procedimiento de necropsia. B) Sección del ápice pulmonar. C) Sección de las regiones posterolaterales del pericardio para la extirpación de los nervios frénicos.

\section{Procesamiento histológico}

Las muestras de tejido se almacenaron en una solución de formaldehído al 10\%, hasta que se procedió al procesamiento histológico convencional para la preparación de las láminas histológicas teñidos con hematoxilina-eosina $(\mathrm{HE})$. Se realizaron 4 láminas histológicas para cada cadáver, uno por cada ápice pulmonar y uno por cada nervio frénico. Las láminas histológicas se analizaron mediante microscopía óptica con objetivos de $4 \mathrm{x}, 10 \mathrm{x}$ y $20 \mathrm{x}$. Los análisis histopatológicos fueron realizados por un patólogo general, que fue evaluador ciego del estudio.

\section{RESULTADOS}

De acuerdo con los criterios de inclusión y exclusión del estudio, se incluyeron 38 cadáveres en la evaluación inicial, de los cuales 31 se incluyeron en el grupo de EPOC y 7 en el grupo de CTR. El perfil sociodemográfico de las muestras de los cadáveres para los grupos CTR y EPOC se puede observar en la Tabla 1. Los cadáveres del grupo EPOC tenían una edad media de 66,8 \pm 17,9 años, mayor prevalencia del género masculino $(61,3 \%, n=19)$, mulato $(64,5 \%, n=20)$ y originario de la capital $(61,3 \%$, $\mathrm{n}=19)$. Como se esperaba, se observó que alrededor del $90 \%(n=28)$ de los cadáveres en el grupo de EPOC tenían antecedentes de tabaquismo. Mientras que los cadáveres del grupo CTR tenían una edad promedio de $73,6 \pm 17,6$ años, mayor prevalencia de mujeres (85,7\%, $\mathrm{n}=6)$, mulato $(85,7 \%, \mathrm{n}=6)$ y origen de la capital $(100 \%$, $\mathrm{n}=7$ ). De acuerdo con el formulario completado por la familia en el SVO, ningún cadáver incluido en el grupo de CTR tenía un historial de tabaquismo. 
Tabla 1. Perfil sociodemográfico de las muestras obtenidas de cadáveres con enfermedad pulmonar Obstructiva Crónica (EPOC) y sin ella (CTR).

\section{$\operatorname{CTR}(n=7)$ \\ EPOC $(n=31)$}

\section{Género}

Femenino

$85,7 \%(6)$

$38,7 \%$

Masculino

$14,3 \%(1)$

$61,3 \%$

Grupo de edad (años)

$73,6 \pm 17,6$

$66,8 \pm 17,9$

\section{Raza}

Blanco

$0 \%$

$32,3 \%$

Mulato

$85,7 \%$

$64,5 \%$

Negro

$14,3 \%$

$3,2 \%$

\section{Procedencia}

Capital

Provincias

\section{Tabaquismo}

$\mathrm{Si}$

$100 \%$
$0 \%$

$61,3 \%$

$38,7 \%$

$100 \%$

No

$100 \%$

$0 \%$

En las muestras de pulmón, el hallazgo histopatológico más notable fue la antracosis presente en todas las muestras del grupo EPOC (Figura 2. B). El grupo EPOC también mostró engrosamiento septal y bullas enfisematosas en el $32,3 \%(n=10)$ y el $22,6 \%(n=7)$ de las muestras pulmonares (Figura 2.CyD, respectivamente). El grupo de CTR no presentó alteraciones histopatológicas de las muestras pulmonares (Figura 2. A).

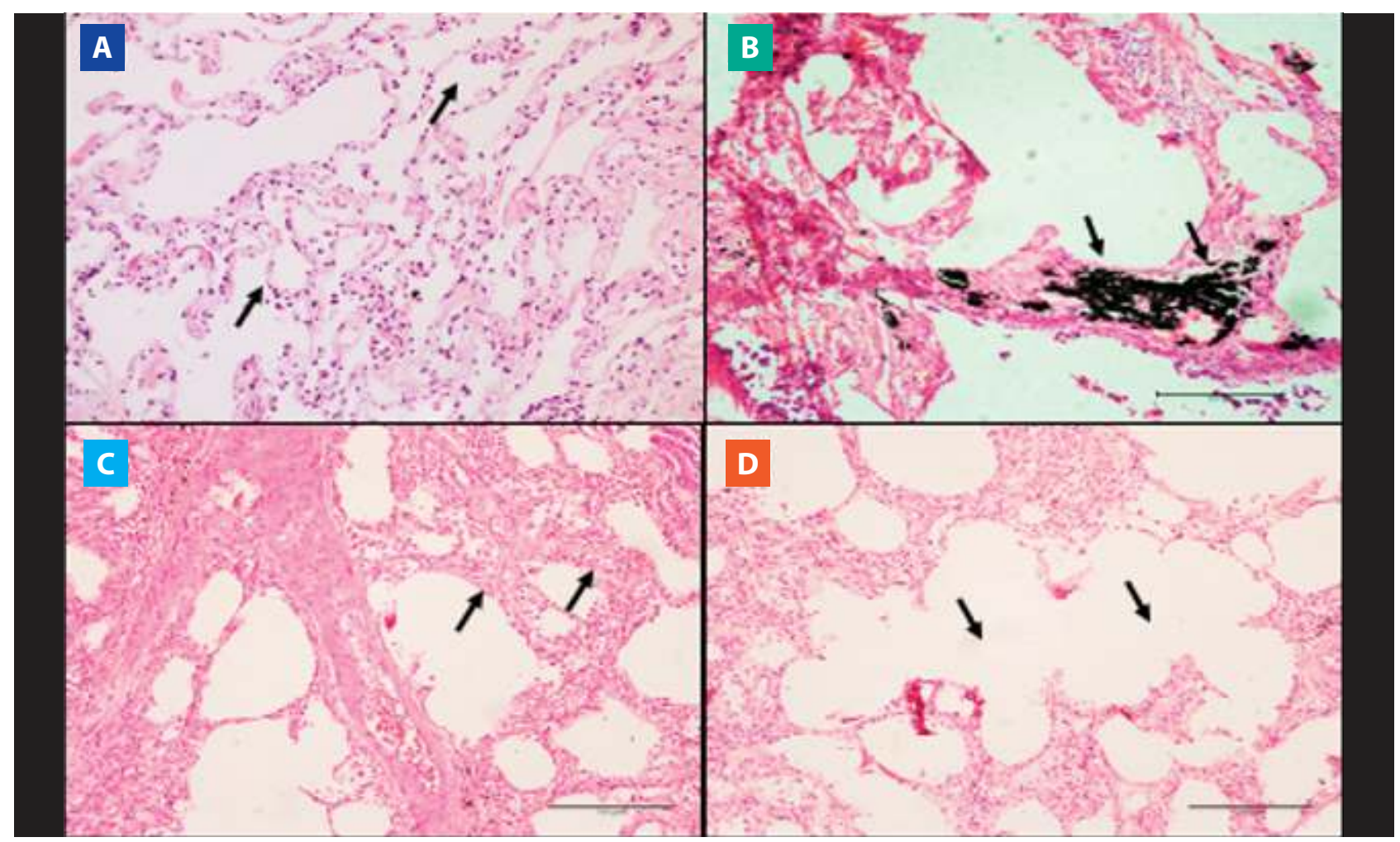

Figura 2. Vista microscópica de tejido pulmonar. Se observan las Imágenes de los cortes histológicos de los vértices pulmonares teñidos con hematoxilina-eosina (HE) en cadáveres con y sin EPOC. A) Morfología pulmonar con septos alveolares y alvéolos preservados (10 aumentos). B) Antracosis pulmonar (aumento 10x). C) Burbujas enfisematosas. (10x aumentos). D) Engrosamiento septal (aumento 10x). 


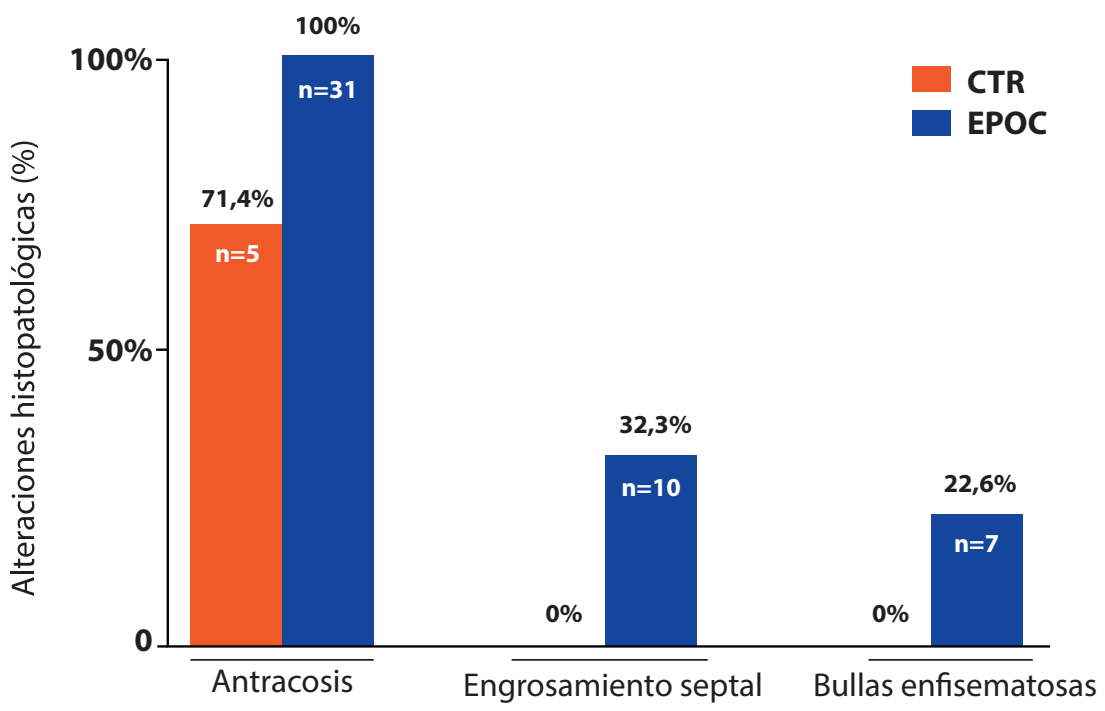

Figura 3. Comparación de hallazgos histopatológicos según grupo de estudio que muestra las alteraciones histopatológicas pulmonares en cadáveres EPOC y CTR.

Con respecto a la evaluación de las muestras de nervios frénicos, 8 cadáveres, es decir, 25,8\%, del grupo de EPOC tuvieron cambios histopatológicos, como edema perineural $(75 \%, n=6$, Figura 4. B), atrofia nerviosa $(12.5 \%, n=1$, Figura 4. C) e infiltrado eosinofílico perineural $(12,5 \%, n=1$, Figura 4. D). El grupo de CTR no presentó alteraciones histopatológicas de los nervios frénicos (Figura 4. A). Es importante destacar que los resultados histopatológicos de los pulmones y los nervios frénicos presentaron el patrón de manera bilateral.

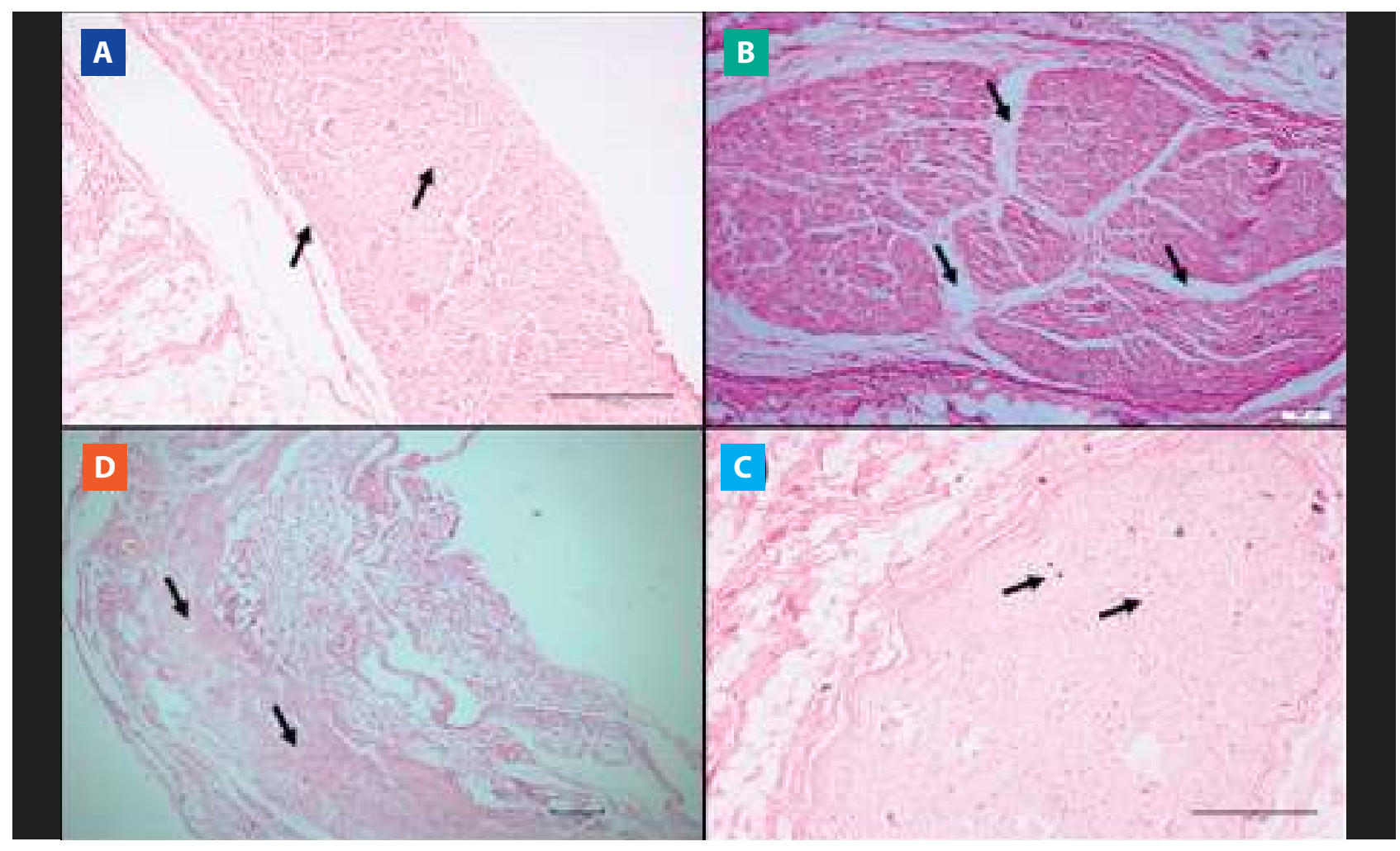

Figura 4. Vista microscópica de tejido nervioso (nervio frénico). Imágenes de láminas histológicas con la técnica de hematoxilina-eosina (HE) en cadáveres con y sin EPOC. A) Morfología del nervio frénico preservado (aumento 10x). B) Edema perineural (aumento 10x). C) Atrofia nerviosa (aumento 10x). D) Infiltrado eosinofílico perineural (aumento de 10x). 


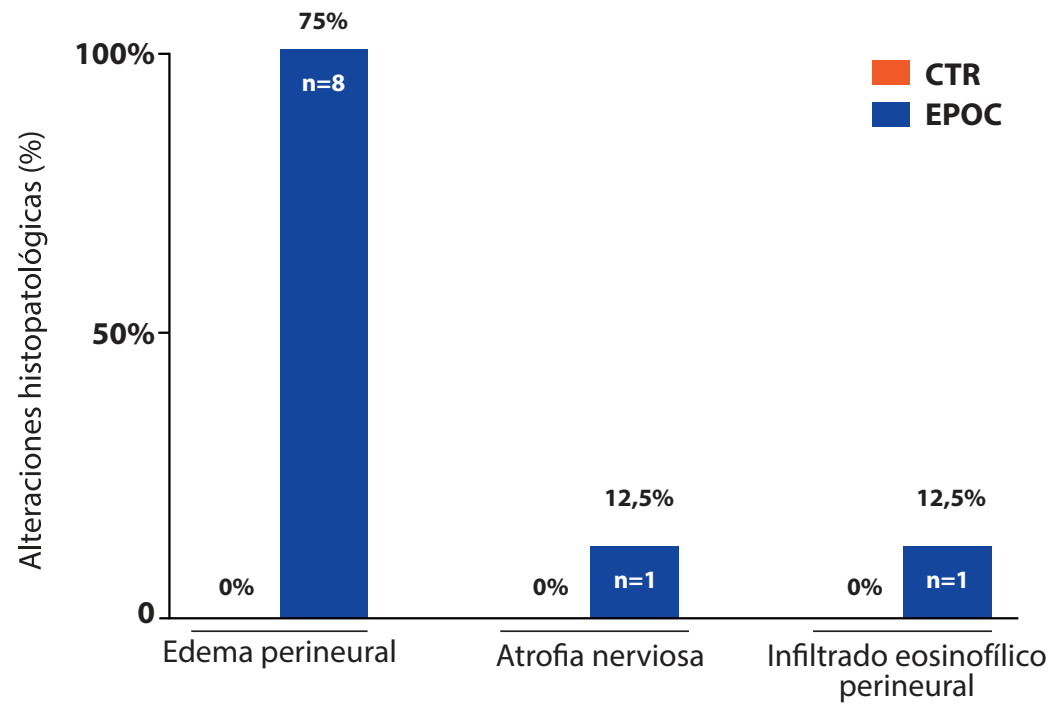

Figura 5. Comparación de hallazgos histopatológicos según grupo de estudio en donde se aprecia las alteraciones histopatológicas de los nervios frénicos en cadáveres con EPOC y CTR.

\section{DISCUSIÓN}

La antracosis se encuentra con frecuencia en individuos con antecedentes de tabaquismo, pero también se puede observar en individuos expuestos a la quema de biomasa utilizado como fuente de energía ${ }^{(11,12)}$. El humo del cigarrillo provoca un reclutamiento grande $y$ continuo de células inflamatorias que liberan enzimas proteolíticas en la matriz extracelular del parénquima pulmonar, causando patrones histopatológicos característicos como engrosamiento septal, agrandamiento de los espacios aéreos, reducción de colágeno y fibras elásticas y aumento de la cantidad de macrófagos alveolares ${ }^{(13)}$. En el presente estudio, nuestro resultado muestra que la antracosis estuvo presente, solo en el grupo de EPOC y la presencia de antracosis en fumadores encontrada en este estudio, corrobora los datos de la literatura, confirmando que el hábito de fumar.

Los estudios han demostrado que el edema perineural se puede visualizar en varias patologías neuronales. El edema perineural proporciona cambios en la estructura neural, ya que desencadena un aumento de la presión tisular del endoneuro, que ocluye las arterias y las venas y produce isquemia ${ }^{(14,15)}$. En este caso en particular, los resultados histopatológicos encontrados en los nervios frénicos del grupo de EPOC, predominó el edema perineural. El edema perineural encontrado en el presente estudio aún permanece oscuro, porque no se encontraron datos consistentes que correlacionaran directamente el edema perineural con el inicio de la EPOC.

El tejido nervioso es poco tolerante al proceso isquémico, por lo que, como mecanismo de compensación, puede producirse un engrosamiento de los nervios. Si el tejido lesionado presenta fibrosis en el epineuro, el engrosamiento del nervio causará rápidamente un aumento de la presión en el compartimento nervioso interno, iniciando un nuevo proceso isquémico ${ }^{(16,17)}$.

Scott y colaboradores, encontraron la presencia de colágeno en la vaina de mielina, así como fibras musculares anormales en los diafragmas de los individuos con EPOC, lo que compromete la generación de fuerza en este músculo respiratorio principal, lo que reduce el rendimiento de la mecánica ventilatoria ${ }^{(18)}$. La adaptación diafragmática existe simultáneamente con el daño al diafragma de un individuo con EPOC, pero esta adaptación no garantiza la misma fuerza y resistencia que se encuentran en el diafragma de un individuo sano ${ }^{(9,19,20)}$

Curiosamente, los estudios han encontrado que los individuos cuadripléjicos pueden tener atrofia del músculo diafragmático después de unos meses de lesión de la médula espinal, reduciendo a la mitad la fuerza contráctil diafragmática, que puede explicarse por la ley de uso y desuso(10,21). A partir de estos datos, es posible inferir que la lesión del nervio motor puede causar alteraciones musculares perjudiciales, y en consecuencia, una función motora deteriorada. Siguiendo el mismo razonamiento, la lesión del nervio motor y la consiguiente denervación de la placa motora promueven la atrofia muscular, afectando preferentemente las fibras de contracción rápida ${ }^{(22)}$. Por lo tanto, aparentemente, existe una asociación entre los cambios encontrados en el diafragma y los nervios frénicos, y un evento puede influir en otro. 


\section{Limitaciones}

Como este fue un estudio pionero, tuvimos dificultades que impidieron un análisis detallado de los cambios histopatológicos encontrados en los nervios frénicos en la condición de EPOC. Entre ellas tenemos a:

- La baja adherencia de los familiares a autorizar el formulario del consentimiento informado (ICF) de los cadáveres para la extracción de tejidos para investigación científica.

- La escasez de referencias bibliográficas existentes en la literatura, lo que restringió la elaboración de discusiones más amplias.

- La técnica histológica utilizada, que fue la convencional.

Por lo tanto, una técnica alternativa para una mejor visualización, identificación y descripción de las estructuras en cuestión, sería la microscopía electrónica asociada con un software adecuado para mediciones.

\section{CONCLUSIÓN}

Dado los resultados de las muestras del presente estudio, los nervios frénicos de los cadáveres afectados por EPOC, presentaron cambios histopatológicos.
También se observó que estos cambios en los nervios frénicos pueden estar relacionados con el desempeño del diafragma; sin embargo, se necesitarán más estudios para probar y explicar estos eventos patológicos y conocer más acerca de los mecanismos fisiopatológicos involucrados en estos procesos, como la aparición de alteraciones musculares y adaptaciones nerviosas.

Contribuciones de autoría: Los autores participaron en la génesis de la idea, diseño de proyecto, recolección e interpretación de datos, análisis de resultados y preparación del manuscrito del presente trabajo de investigación.

\section{Financiamiento: Autofinanciado.}

Conflicto de interés: Los autores declaran no tener conflicto de interés en la publicación de este artículo.

Recibido: 01 de febrero 2020

Aprobado: 10 de marzo 2020

\section{Correspondencia: Tales Lyra de Oliveira.}

Dirección: USCS, Rua Treze de Maio, 681, Bela Vista, CEP 01327-000, São Paulo - SP - Brasil.

Teléfono: $+551195222-4180$

Correo: tales.oliveira@prof.uscs.edu.br

\section{REFERENCIAS BIBLIOGRÁFICAS}

1. Lozano R, Naghavi M, Foreman K, Lim S, Shibuya K, Aboyans V, et al. Global and regional mortality from 235 causes of death for 20 age groups in 1990 and 2010: a systematic analysis for the Global Burden of Disease Study 2010 Lancet. 2012;380(9859):2095-2128. DOI: 10.1016/S0140-6736(12)61728-0.

2. Mathers $C D$, Loncar D. Projections of global mortality and burden of disease from 2002 to 2030. PLoS Med. 2006;3(11):2012-2030. DOI: 10.1371/ journal.pmed.0030442.

3. Singh D, Agusti A, Anzueto A, Barnes PJ, Bourbeau J, Celli BR, et al Global Strategy for the Diagnosis, Management, and Prevention of Chronic Obstructive Lung Disease: the GOLD science committee report 2019. Eur Respir J. 2019;53(5): 1 - 23. DOI: 10.1183/13993003.00164-2019

4. Lange $P$, Celli B, Agustí A, Boje Jensen G, Divo M, Faner R, et al. Lung Function Trajectories Leading to Chronic Obstructive Pulmonary Disease. N Engl J Med. 2015;373(2):111-122. DOI: 10.1056/NEJMoa1411532.

5. Alter A, Aboussouan LS, Mireles-Cabodevila E. Neuromuscular weakness in chronic obstructive pulmonary disease: chest wall, diaphragm, and peripheral muscle contributions. Curr Opin Pulm Med. 2017;23(2):129-138 DOI: $10.1097 / M C P .0000000000000360$.

6. Negewo NA, Gibson PG, McDonald VM. COPD and its comorbidities: Impact, measurement and mechanisms. Respirology. 2015;20(8):1160-1171. DOI: $10.1111 /$ resp. 12642 .

7. Naruse $M$, Ishizaki $Y$, Ikenaka $K$, Tanaka A, Hitoshi S. Origin of oligodendrocytes in mammalian forebrains: a revised perspective. J Physiol Sci. 2017;67(1):63-70. DOI: 10.1007/s12576-016-0479-7.

8. Bacci A, Verderio C, Pravettoni E, Matteoli M. The role of glial cells in synaptic function. Philos Trans R Soc Lond B Biol Sci. 1999;354(1381):403-409. DOI: $10.1098 /$ rstb.1999.0393.

9. Ramírez-Sarmiento A, Orozco-Levi $M$, Barreiro $E$, Méndez R, Ferrer A Broquetas J, et al. Expiratory muscle endurance in chronic obstructive pulmonary disease.Thorax.2002;57(2):132-136.DOI: 10.1136/thorax.57.2.132

10. Garara B, Wood A, Marcus HJ, Tsang K, Wilson MH, Khan M. Intramuscular diaphragmatic stimulation for patients with traumatic high cervical injuries and ventilator dependent respiratory failure: A systematic review of safety and effectiveness. Injury. 2016;47(3):539-544. DOI: 10.1016/j.injury.2015.12.020.

11. Martinelli LM, Boas PJ, Queluz TT, Yoo HH. Morphological prognostic factors in nosocomial pneumonia: an autopsy study. J Bras Pneumol.

\section{0;36(1):51-58. DOI: 10.1590/s1806-37132010000100010.}

12. Churg A, Brauer M, del Carmen Avila-Casado M, Fortoul TI, Wright JL. Chronic exposure to high levels of particulate air pollution and small airway remodeling. Environ Health Perspect. 2003;111(5):714-718. DOI: 10.1289/ ehp.6042.

13. Lagente V, Manoury B, Nénan S, Le Quément C, Martin-Chouly C, Boichot E. Role of matrix metalloproteinases in the development of airway inflammation and remodeling. Braz J Med Biol Res. 2005;38(10):1521-1530. DOI: $10.1590 / \mathrm{s} 0100-879 \times 2005001000009$.

14. Nations SP, Katz JS, Lyde CB, Barohn RJ. Leprous neuropathy: an American perspective. Semin Neurol. 1998;18(1):113-124. DOI: 10.1055/s-20081040867.

15. Antia NH. The significance of nerve involvement in leprosy. Plast Reconstr Surg. 1974;54(1):55-63. DOI: 10.1097/00006534-197407000-00007.

16. Walker SL, Lockwood DN. Leprosy type 1 (reversal) reactions and their management. Lepr Rev. 2008;79(4):372-386. Disponible en: https://www. lepra.org.uk/platforms/lepra/files/Ir/Dec08/Lep372-386.pdf.

17. Forgione P, Barabino G, Cavalchini A, Clapasson A, Reni L, Parodi A Pure neuritic leprosy. G Ital Dermatol Venereol. 2018;153(1):124-126. DOI: 10.23736/S0392-0488.16.05474-2.

18. Scott A, Wang X, Road JD, Reid WD. Increased injury and intramuscular collagen of the diaphragm in COPD: autopsy observations. Eur Respir J. 2006;27(1):51-59. DOI: 10.1183/09031936.06.00143004

19. Orozco-Levi M. Structure and function of the respiratory muscles in patients with COPD: impairment or adaptation? Eur Respir J Suppl. 2003;46:41-51. DOI: 10.1183/09031936.03.00004607.

20. Levine S, Gregory C, Nguyen T, Shrager J, Kaiser L, Rubinstein N, et al. Bioenergetic adaptation of individual human diaphragmatic myofibers to severe COPD. J Appl Physiol (1985). 2002;92(3):1205-1213. DOI: 10.1152/ japplphysiol.00116.2001.

21. DiMarco AF. Diaphragm Pacing. Clin Chest Med. 2018;39(2):459-471. DOI: 10.1016/j.ccm.2018.01.008.

22. Proctor DN, Balagopal P, Nair KS. Age-related sarcopenia in humans is associated with reduced synthetic rates of specific muscle proteins. J Nutr. 1998;128(2):351-355. DOI: 10.1093/jn/128.2.351S 